\title{
Contributions of Elastically and Inelastically Scattered Electrons to High-Resolution Off-Axis Electron Holograms - a Quantitative Analysis
}

\author{
Michael Lehmann and Hannes Lichte \\ University of Dresden, Institute of Applied Physics (IAPD), D-01062 Dresden, Germany
}

Off-axis electron holograms incorporate not only the information of conventional image intensity but also the perfect zero-loss information of the object wave encoded in the interference fringes. This is due to the setup of off-axis electron holography: Since the reference wave passes the object plane unscattered, only electrons which are elastically scattered in the object are still coherent hence can contribute to the interference pattern. For coherent detection, the energy loss in the object must be less than $4 \cdot 10^{-15} \mathrm{eV}$, which is an energy width far beyond the possibilities of current energy filters. After Fourier transformation of the hologram, the information of the conventional HRTEM image including inelastics is found in the centerband whereas the perfect zero-loss information of the complex object wave is well-separated in the sidebands; therefore, off-axis electron holography allows a quantitative comparison at exactly the same specimen area. The conventional HRTEM image is gained by isolation of the centerband and inverse Fourier transformation. Centering and isolating one sideband, inversely Fourier transforming, and calculating the intensity of the image wave yields the perfect zero-loss image intensity. The normalization of both image intensities is normally performed at an image area without object. If this area is not available within the field of view, the reference hologram can be used due to the high stability of both microscope and Triebenberg lab hence high constancy of fringe contrast over nearly all exposures.

Fig. 1 (top) shows the image intensities of a 90-degree GaAs-wedge in [100]-orientation reconstructed from centerband and sideband, respectively. The image intensity reconstructed from the sideband shows a higher contrast than the other one. However, due to different noise contributions to centerband and sideband, contrast determination of lattice fringes is much more reliable when calculated from nano-diffractograms (fig. 1 bottom) by division of intensity of reflections by the zero-beam intensity. The ratio of corresponding lattice fringe contrasts in sideband and centerband reconstructed image intensity, respectively, is plotted over the sample thickness (fig. 2). With increasing sample thickness beyond the first extinction thickness, the plot shows an up to 4.3 times higher lattice fringe contrast in the zero-loss image. The drop of contrast at subimages 5 and 6 is probably due to increasing noise in the sideband. Recently, a series of off-axis electron holograms taken at different defocus has been recorded and analyzed showing nearly the same lattice fringe contrasts in amplitude and phase when reconstructed from sideband but less lattice fringe contrast when the focal-series is reconstructed from centerband [1].

Making use of both centerband and sideband information suggest that the contrast mismatch between simulated and experimental HRTEM image intensities, often referred to as "StobbsFactor", is mainly due to contributions from inelastically scattered electrons. Since the lattice fringe contrast reconstructed from sideband is substantially larger compared with contrasts of HRTEM images, we assume that off-axis electron holography is only less affected by the Stobbs-Factor.

[1] M. Lehmann, D. Geiger, I. Büscher, H.W. Zandbergen, D. Van Dyck, H. Lichte, submitted to ICEM-15 (2002), Durban, South Africa 

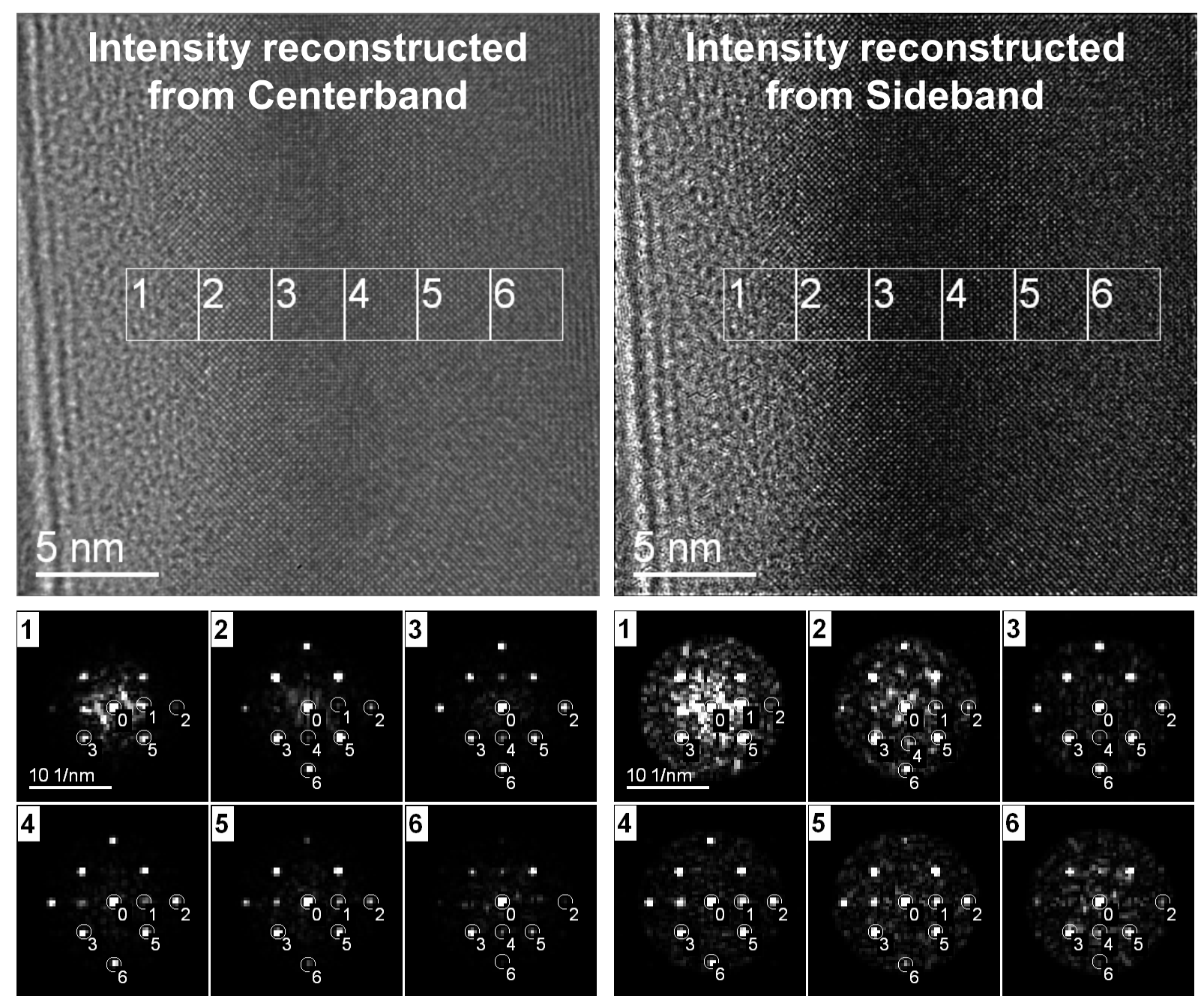

Fig. 1 top: image intensity (display range 0 .. 2) of a 90-degree GaAs-wedge in [100]-orientation reconstructed from centerband (left) and sideband (right), respectively. Bottom: nanodiffractograms determined from subimages of the image intensities shown above. The increasing subimage number corresponds to increasing sample thickness.

Fig. 2: Contrast mismatch of lattice fringes reconstructed from sideband and centerband, ISB and ICB, respectively, plotted over the subimage number i.e. the sample thickness. The sample thickness increases from left to right. Subimages 3 and 6 correspond to the first and second extinction thickness.

Thanks are due to Ides Büscher and Henny W. Zandbergen not only for providing the GaAs-wedge sample but also, together with Dirk Van Dyck, for the close cooperation.

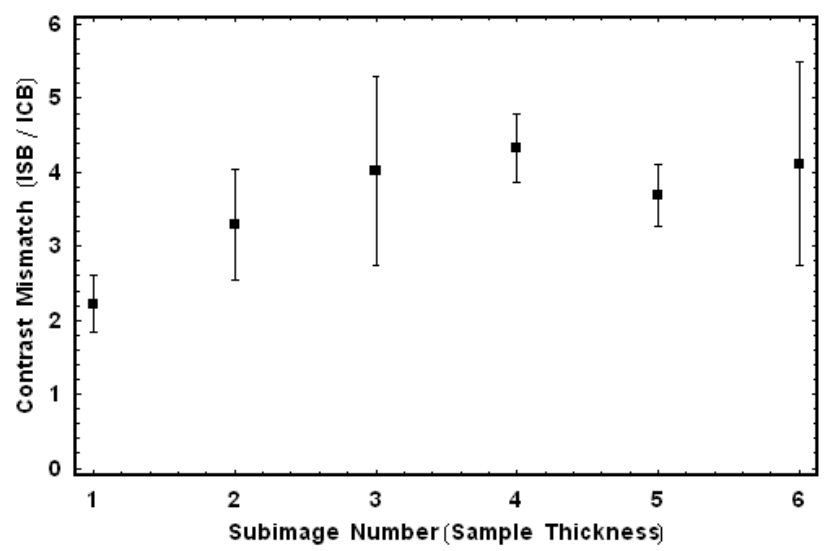
The financial support by the DFG within the framework of SFB 422 is gratefully acknowledged. 